\title{
The Influence of the Most Powerful Signals on the Pacing Site Localization by Single Dipole
}

\author{
Jana Svehlikova ${ }^{1}$, Beata Ondrusova ${ }^{1}$, Jan Zelinka $^{1}$, Milan Tysler ${ }^{1}$ \\ ${ }^{1}$ Institute of Measurement Science, SAS, Bratislava, Slovakia
}

\begin{abstract}
In real experiments with body surface potential mapping, it is usual that the measuring electrodes cannot be placed regularly because of other patches of sensors used on the torso during the procedure. The influence of accidentally missing the most powerful ECG signals on the inverse localization of the pacing site using a single dipole was studied.

Body surface potential maps from three tank experiments on the pig or dog heart and one measurement from the patientôs torso were used for inverse computation of the known pacing site position using the full electrodes setup and setups omitting $10 \%$ and $20 \%$ of the signals with the largest power. The sensitivity of the inverse method was evaluated by localization error between the true and computed pacing site.

The localization error did not change (13mm or $7 \mathrm{~mm})$ or changed from 13 to $24 \mathrm{~mm}$ for tank data, but it increased rapidly from 19 to $41 / 68 \mathrm{~mm}$ for human data.

The largest effect of electrodesôomission using human data can be explained by the largest complexity of the human torso what is not fully included in the computational torso model.
\end{abstract}

\section{Introduction}

The electrocardiographic imaging is based on a multiple-leads ECG measurement on the torso. Usually, the proposed arrangement of electrodes is a regular grid around the patient's chest. If an irregular placement of electrodes is used the highest density of electrodes occurs on the anterior torso where the largest spatial gradient of the measured electrical field is expected. However, in real situations when the body surface potential (BSP) mapping is performed along with another procedure, the areas for defibrillation patches or CARTO patches have to be left free of measuring electrodes. The variability of the measuring electrode placement during the real measurements can be seen e.g. in [1]. Also, sometimes, after several minutesô preparation of the patient some broken electrodes are found just when all electrodes are placed on the torso.

The problem of missing or small amplitude signals is being solved also in the Signal preprocessing group of the Consortium of Electrocardiographic Imaging. The novel method for interpolation of missing signals was introduced in [2]. The effect of the interpolation of ECG signals on the results of the inverse solution (ECG imaging) for various scenarios of omitted electrodes was studied in [3] and [4]. However, according to the published results, the interpolation of the missing signals did not improve the results in terms of inversely reconstructed electrograms, thus we need to rely only on the measured signals.

Theoretically, the inverse solution using a single dipole heart model does not need many measured signals on the torso. In [5] it was shown that the small noisy signals do not influence the results of this inverse method. Therefore, in this study, we investigated the situation when accidentally just the signals with the largest power are not available.

\section{Method and Materials}

Multiple leads ECG measurements from four experiments using three different heart-torso setups were used in the study. Bordeaux data (provided by IHU_LIRYC Bordeaux, France) were obtained from pig heart inserted in a homogeneous torso tank measured in 128 electrodes on the tank surface [6]. Utah data (provided by SCI Salt Lake City, Utah) from two experiments were measured from dog heart inserted in a homogeneous torso tank with 192 electrodes placed regularly in $12 \times 16$ mesh around the torso model [7]. The data were available within cooperation in the Signal processing group from Consortium for ECGI (https://www.ecgimaging.org/workgroups/signal-processing). Last data set (human data) was from body surface potential mapping of a patient measured in 128 electrodes located in an $8 \times 16$ grid on the torso. The torso model of the patient was created from a CT scan. The data were obtained within a collaboration project of the Department of Biomeasurements, IMS SAS and the National Institute for Cardiovascular Diseases, Bratislava, Slovakia [8].

In all experiments, the heart activity was stimulated in 
the ventricles by a single electrode.

From the measured body surface potential maps (BSPMs), the position of the stimulating electrode was computed by the inverse method using a single dipole as an equivalent electrical heart generator [9]. In this method, the very early time interval of depolarization is considered (up to $25 \mathrm{~ms}$ ). It is assumed that during this time the activated area is small enough to be represented by a single dipole. The best localization of the representative dipole is searched in the ventricular volume in the defined positions of nodes. Let $\mathbf{T}$ be a transfer matrix between the heart dipole $\mathrm{d}$ in a particular position and the torso potentials $\mathrm{p}$. Then the potentials can be computed as:

$\mathrm{p}=\mathbf{T d}$

From the known potential map, the unknown dipole moments can be obtained by solution of the overdetermined system of linear equations:

$\mathrm{d}=\mathbf{T}^{+} \mathrm{p}$

where $\mathbf{T}^{+}$is a pseudoinverse of the transfer matrix.

All available signals from each experiment were processed as follows. First, a baseline drift (BDR) was removed by fitting the determined zero points by a cubic spline. To eliminate the high-frequency noise, the ECG signal was segmented according to its repeated morphology and averaged. The averaged BSPMs were used as input data for the inverse computation.

In each measuring electrode, the power of the signal during the first $25 \mathrm{~ms}$ time interval from the beginning of depolarization was computed as a sum of the squares of the signal amplitudes in each sampling point. The measuring electrodes were sorted according to the signal power value in descending order.

The signal-to-noise ratio (SNR) during the studied time interval was also evaluated for each electrode in each time sample $\mathrm{t}$ according to the formula:

$\operatorname{SNR}(t)=20 \log 10($ mean_sig $(t) /$ std_sig $(t)$

where mean_sig $(t)$ was the averaged signal in a given time instant $t$ and std_sig $(t)$ was the standard deviation of the values used for the mean computation.

The position of the stimulating electrode was known for each experiment. First, the localization of the stimulating electrode was computed for the full set of electrodes. A localization error (LE) was calculated as the Euclidean distance between the true position of the electrode and the position of the dipole computed by the inverse solution. Then, $10 \%$ and $20 \%$ of the measuring electrodes with the largest value of the signal power during the first $25 \mathrm{~ms}$ were removed from the data. The influence of such removal on the LE was evaluated for each experiment.

\section{Results}

The power of the signals during the first $25 \mathrm{~ms}$ of the QRS interval arranged in descending order for each experiment is displayed in graphs in Figure 1.

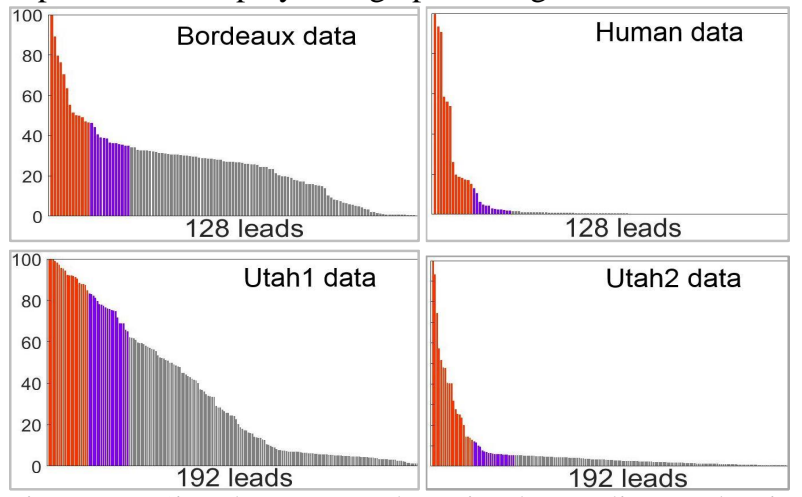

Figure 1. Signal power values in descending order in measuring electrodes computed during the first $25 \mathrm{~ms}$ of depolarization for four experiments. Vertical axis assigns $100 \%$ for the lead with maximal power. Red: $10 \%$ of leads, red+violet: $20 \%$ of leads omitted in the study.

The torso models with the positions of electrodes removed in the study are depicted in Figure 2.

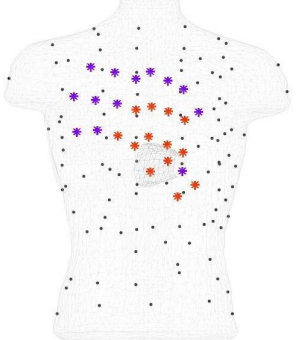

Bordeaux

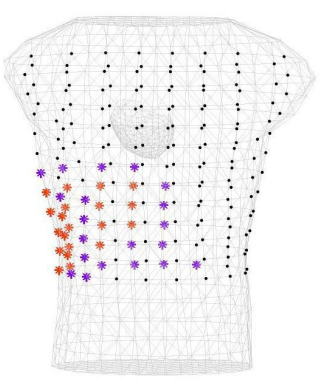

Utah1

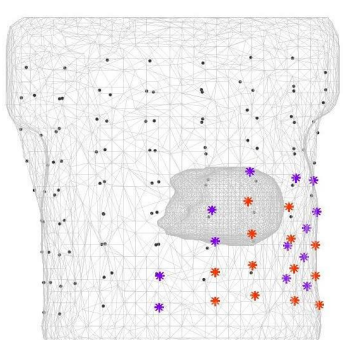

Human

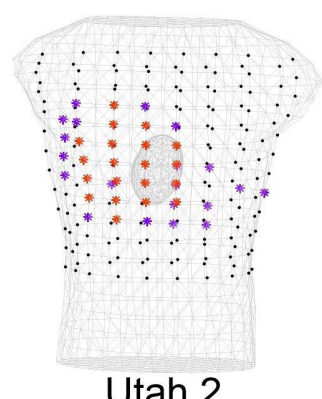

Utah 2
Figure 2. Measuring electrodes in four experiments (Bordeaux, Human, Utah1, Utah2) with 10\% (red) and $20 \%$ (red + violet) of the electrodes with most powerful signals.

Maximal SNR values within the first $25 \mathrm{~ms}$ of depolarization for the electrodes ordered according to the signal power values corresponding to Figure 1 are depicted in Figure 3. The number of heartbeats used for the 
averaging was 28 for the Bordeaux data, 480 for the human data and 15 or 19 beats for the Utah1 or Utah2 data, respectively. The value of maximal SNR within the first $25 \mathrm{~ms}$ of depolarization for each experiment is shown in Table 1 .

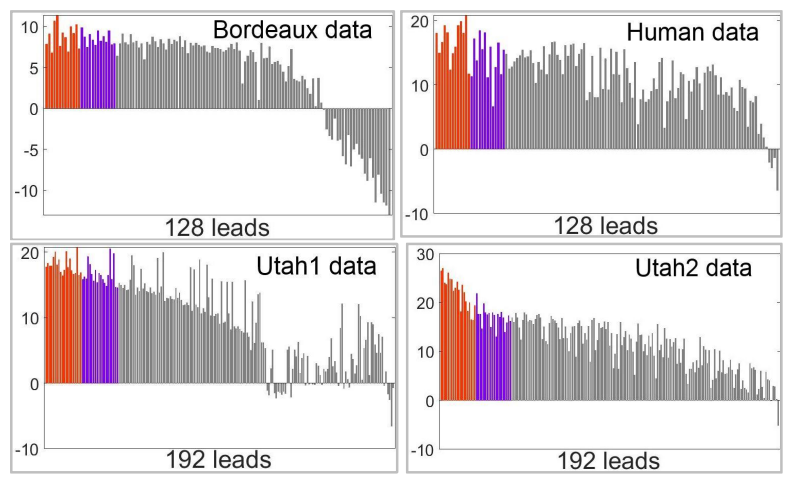

Figure 3. Maximal SNR values in $[\mathrm{dB}]$ for the electrodes ordered according to the highest signal power value for each experiment. Colors correspond to Figure 1.

For torso models with 128 electrodes, 13 (10\%) and 26 (20\%) leads with maximal signal power value were removed, for models with 192 leads, 20 and 40 leads were omitted accordingly.

To make the results from all four experiments better comparable, the number of electrodes for Utah torso model was reduced by 64 electrodes to have the same number of electrodes for all experiments. The 64 electrodes were removed in such a way that the electrodes with the largest signal power remained among the used 128 leads. Therefore, for Utah1 the four most upper rows of electrodes were removed, while for Utah2 the two most upper and two lowest rows of electrodes were removed. From the remaining 128 electrodes again 13 and 26 leads were omitted similarly as for the Bordeaux and human data.

The position of the stimulating electrode was computed by the inverse solution to one dipole for all mentioned configurations of electrodes. The obtained LEs are presented in Table 1.

Table 1. Maximal SNR within the first $25 \mathrm{~ms}$ of depolarization. LEs for all studied configurations of measuring electrodes.

\begin{tabular}{lcccc}
\hline & \multicolumn{4}{c}{ LE [mm] } \\
\hline Experiment & $\begin{array}{c}\text { maxSNR } \\
{[\mathrm{dB}]}\end{array}$ & $\begin{array}{c}100 \% \\
\text { of leads }\end{array}$ & $\begin{array}{c}90 \% \\
\text { of leads }\end{array}$ & $\begin{array}{c}80 \% \\
\text { of leads }\end{array}$ \\
\hline Bordeaux & 11.4 & 12.9 & $\mathbf{2 3 . 8}$ & 15.0 \\
Human-homog. & 20.9 & $\mathbf{1 9 . 2}$ & $\mathbf{4 1 . 6}$ & $\mathbf{6 8 . 2}$ \\
Human-inhomog. & & 15.0 & 31.0 & 61.4 \\
Utah1-192 leads & 20.8 & 13.3 & 13.3 & 13.3 \\
Utah1ï 128 leads & & 13.4 & 13.4 & 13.4 \\
Utah2-192 leads & 27.1 & 6.9 & 6.9 & 6.9 \\
Utah2-128 leads & & 6.9 & 6.9 & 6.9 \\
\hline
\end{tabular}

\section{Discussion}

From the graphs in Figure 1, it is visible that the number and magnitude of the large power values differed considerably from case to case. The drop of the power value was from $15 \%$ for Utah1 to $87 \%$ for Utah2 data when $10 \%$ of the most powerful electrodes were considered, and from $35 \%$ for Utah1 to $98 \%$ for Human data within $20 \%$ of the electrodes with the largest power.

From Figure 2, for the Bordeaux data, electrodes with the most powerful signals were situated anteriorly and with slight prevalence on the right side, on the human torso, such electrodes were on the lower left anterior chest. For Utah1 data, the electrodes with the largest signal power during the first $25 \mathrm{~ms}$ were surprisingly on the lower lateral and posterior right side, while for Utah2 data such electrodes were on two opposite sides on the torso $\ddot{i}$ anterior slightly right and posterior. This wide variability resulted from the different positioning of the isolated animal hearts in the torso tanks as it is apparent from the Figure 2, from the variability of the location of the pacing sites in the ventricles, as well as from the specific short time interval (starting $25 \mathrm{~ms}$ ) when the signal power in the electrodes was computed. When the signal power in the electrodes was computed from the whole QRS complex the positions of electrodes with the most powerful signals appeared anteriorly (as it is generally expected) and did not differ too much (Figure 4).
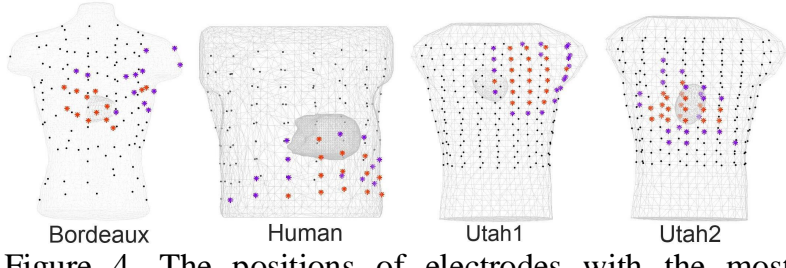

Figure 4. The positions of electrodes with the most powerful signals on the torso if the power was computed from the whole QRS time interval. Colors correspond to Figure 2.

No sensitivity to electrode removal was observed for both Utah data sets when the LE remained on the very agreeable value of 13 and $7 \mathrm{~mm}$, respectively, even if the original arrangement of 192 electrodes was reduced by 64 electrodes. However, the reduction from 192 to 128 electrodes was performed by maintaining the largest signals, what was just one of many other possibilities on how to perform it. The absence of the signals with small amplitudes did not worsen the LE.

The largest LE for the Bordeaux data was observed when $10 \%$ of the most powerful signals were omitted, the absence of $20 \%$ of the most powerful signals surprisingly improved the LE. It points out that the power and number of signals are not the only important parameters for good results. Thus, other parameters need to be studied in more details in a larger study. 
The largest sensitivity to the absence of electrodes with the most powerful signals was observed in human data for which just the absence of $10 \%$ of electrodes lead to the LE greater than $30 \mathrm{~mm}$.

The SNR values from Figure 3 did not change so rapidly as the power of the signals in Figure 1, SNR remained similar for more than half of the measured signals. After omitting $20 \%$ of the signals there were still enough signals left with a proper SNR to provide input information for an inverse solution. However, even though the SNR of the signals from the human data was comparable with SNR values of the Utah1 data, the LE increased considerably with the number of omitted electrodes.

There can be two possible reasons for the worst results for the human data. First, more than $99 \%$ of the whole signal power was in the omitted signals. The second reason can be the complexity of the real torso in comparison to the computational torso model. While the computational tank models corresponded well with the true ones for the Bordeaux and Utah data (in both cases the models were homogeneous), for human data the torso models did not represent the reality so truly. Inclusion of some inhomogeneities in the human torso model slightly improved the results, what confirms also the findings in [10] where a positive effect of the inclusion of torso inhomogeneities on the ECGI inverse solution was presented.

\section{Conclusions}

The pacing site localization using a single dipole was not strongly affected for data from tank experiments even if the drop of the power in the omitted signals was more than $90 \%$. However, for real human data, the method was more sensitive to the absence of large signals. It seems that besides the lack of the powerful signals, the important impact on the results had the quality of the torso model. For Bordeaux and Utah data the torso models corresponded with the reality (homogeneous torso tank). For human data, the torso model was always just an approximation of the reality (worse or better, homogeneous or inhomogeneous, respectively) thus the large signals were crucial for obtaining correct results even for a heart generator model as simple as a dipole.

Although the inverse method using a single dipole theoretically does not need many signals, in real measurements it is important not to lose large areas of mapped potentials because it can be essential signal information there.

\section{Acknowledgments}

The study was inspired and the data were provided by the ECGI Signal Preprocessing/Interpolation Group.

This work was supported by the research grant
2/0125/19 from the VEGA Grant Agency in Slovakia and by the grant APVV-19-0531 from the Slovak Research and Development Agency.

\section{References}

[1] Schulze WHW. ECG Imaging of Ventricular Activity in Clinical Applications. PhD Thesis. Karlsruhe: KIT Scientific Publishing, Karlsruhe; 2015.

[2] Rababah Msc AS, Bond RR, Msc KR, Guldenring D, McLaughlin J, Finlay DD. Novel hybrid method for interpolating missing information in body surface potential maps. J Electrocardiol 2019;57:S51ї 5. doi:10.1016/j.jelectrocard.2019.09.003.

[3] Bear L, Potse M, Duchateau J, Zemzemi N, Coudière Y, Dubois R. Accuracy of lead removal vs linear interpolation in non-invasive electrocardiographic imaging (ECGI). Comput Cardiol (2010) 2015; 42:941ї 4. doi:10.1109/CIC.2015.7411067.

[4] Dogrusoz YS, Bear LR, Bergquist J, Dubois R, Good W, Macleod RS, et al. Effects of Interpolation on the Inverse Problem of Electrocardiography. Comput. Cardiol. (2010)., vol. 46, 2019, p. 10ї 3. doi:10.22489/CinC.2019.100.

[5] Deutsch E, Svehlikova J, Tysler M, Osmancik P, Zdarska J, Kneppo P. Effect of elimination of noisy ECG leads on the noninvasive localization of the focus of premature ventricular complexes. IFMBE Proc., vol. 68, Springer Verlag; 2019, p. 75ï 9. doi:10.1007/978-981-10-9035-6_14.

[6] Bear LR, Huntjens PR, Walton RD, Bernus O, Coronel R, Dubois R. Cardiac electrical dyssynchrony is accurately detected by noninvasive electrocardiographic imaging. Heart Rhythm 2018; doi:10.1016/j.hrthm.2018.02.024.

[7] Shome S, Macleod R. Simultaneous high-resolution electrical imaging of endocardial, epicardial and torso-tank surfaces under varying cardiac metabolic load and coronary flow. Lect Notes Comput Sci (Including Subser Lect Notes Artif Intell Lect Notes Bioinformatics) 2007;4466 LNCS:320ї 9. doi:10.1007/978-3-540-72907-5 33.

[8] Svehlikova J, Zelinka J, Haska M, Tysler M. Simulation of measured body surface potential map during early right ventricular pacing. 2019 Proc. 12th Int. Conf. Meas. Meas. 2019, Institute of Electrical and Electronics Engineers Inc.; 2019 , p. $34 \ddot{1} 7$.

[9] Svehlikova J, Teplan M, Tysler M. Geometrical constraint of sources in noninvasive localization of premature ventricular contractions. J Electrocardiol 2018;51:370ї 7. doi:10.1016/j.jelectrocard.2018.02.013.

[10]Zemzemi N, Dobrzynski C, Bear LR, Potse M, Dallet C, Coudiere $\mathrm{Y}$, et al. Effect of the torso conductivity heterogeneities on the ECGI inverse problem solution. Comput. Cardiol. Conf., vol. 42, IEEE; 2015, p. 233 ï 6. doi:10.1109/CIC.2015.7408629.

Address for correspondence:

Jana Svehlikova

Institute of Measurement Science, Slovak Academy of Sciences Dubravska cesta 9, 84104 Bratislava, Slovakia

jana.svehlikova@savba.sk 